\title{
Féeries
}

Études sur le conte merveilleuX, XVII $-\mathrm{XIX}{ }^{\mathrm{e}}$ siècle

\section{De l'allée du Roi aux sentiers du bon sauvage}

Un parcours dans les contes de Madame d'Aulnoy

\section{Marie-Agnès Thirard}

\section{(2) OpenEdition}

Journals

Édition électronique

URL : http://journals.openedition.org/feeries/142

DOI : $10.4000 /$ feeries. 142

ISSN : 1957-7753

Éditeur

UGA Éditions/Université Grenoble Alpes

Édition imprimée

Date de publication : 1 février 2006

Pagination : 59-74

ISBN : 2-84370-082-8

ISSN : $1766-2842$

Référence électronique

Marie-Agnès Thirard, «De l'allée du Roi aux sentiers du bon sauvage », Féeries [En ligne], 3 | 2006, mis en ligne le 05 février 2007, consulté le 08 septembre 2020. URL : http://journals.openedition.org/

feeries/142; DOI : https://doi.org/10.4000/feeries.142

Ce document a été généré automatiquement le 8 septembre 2020.

(c) Féeries 


\title{
De l'allée du Roi aux sentiers du bon
} sauvage

Un parcours dans les contes de Madame d'Aulnoy

\author{
Marie-Agnès Thirard
}

1 LeS FIGURES DU POUVOIR sont présentes dans l'univers des contes populaires et le personnage du roi correspond à un rôle narratif qui fait figure de topos. L'ascension sociale du héros passe le plus souvent par le subterfuge du mariage royal que les études de Propp répertoriaient déjà dans les trente et une fonctions au rang des invariants des récits merveilleux. C'est dire qu'au cœur même des contes populaires, pouvoir et royauté occupent une place de choix. Mais dans le pays de la merveille, il existe un royaume particulier, celui des féeries et une reine, $\mathrm{M}^{\mathrm{me}} \mathrm{d}$ 'Aulnoy. C'est elle qui fut en effet, à la fin du $\mathrm{XVII}^{\mathrm{e}}$ siècle, l'initiatrice de cette mode des récits féeriques, sorte d'exception culturelle à la française dont l'onde de choc se propagea ensuite sur toute l'Europe jusqu'à la fin du XviII èècle avant de venir s'échouer sur les rivages de la littérature de jeunesse et de la littérature de colportage. Le temps n'est plus où le seul nom de Perrault suffisait à rendre compte de ce phénomène littéraire. L'œuvre des autres conteurs et surtout des conteuses surgit enfin des oubliettes de l'histoire littéraire et suscite un nouvel engouement au point de faire l'objet de recherches universitaires et de rééditions ${ }^{1}$. Or Mme d'Aulnoy, en récupérant un genre narratif alors relégué dans la bouche des mères l'Oie devait se situer par rapport à la tradition et transformer l'héritage pour le rendre acceptable à un nouveau lectorat, celui des mondains et des lettrés vivant dans l'orbite de la Cour de Versailles. Il est donc intéressant de cerner les signes de cette métamorphose à travers le prisme de l'image de la royauté et du pouvoir. L'aventure est d'autant plus passionnante que cette mode des contes de fées répond à un besoin de conquête du pouvoir de l'écriture décliné au féminin et que cette mode des contes se situe à la fin d'un règne, celui du Roi-Soleil, roi absolu de droit divin qui prétendait réglementer aussi l'univers culturel. Dans le labyrinthe des deux recueils de notre conteuse que constituent Les Contes des fées et Les Contes nouveaux ou les Fées à la mode, un fil d'Ariane politique pourrait nous guider de l'allée du roi jusqu'au sentier du Bon Sauvage en passant par l'insula feminarum. 
2 Les contes de Mme d'Aulnoy se présentent à un premier niveau de lecture comme le miroir valorisant de toute une société inégalitaire vivant sous les lambris dorés et dans les sphères du pouvoir. La reine de la féerie paraît participer à une opération de propagande à l'égard du pouvoir en place. On se souvient de la mise en scène que Mme d'Aulnoy fait d'elle-même dans le récit-cadre au premier niveau des contes. La conteuse se présente en effet sous la forme d'un personnage qui se promène dans le parc de Saint-Cloud avec des amis et reste seule un moment à l'écart des autres. Durant ce temps de solitude, elle reçoit la visite de la nymphe de Saint-Cloud qui lui transmet des vers élogieux à propos des lieux où elle se trouve. Le château de Saint-Cloud était alors résidence royale. Il était habité par le duc d'Orléans, frère du roi et par la princesse Palatine, son épouse, à qui notre conteuse dédie d'ailleurs des vers élogieux inspirés par la nymphe. Or le pseudonyme de Madame D. attribué au personnagenarrateur correspond à celui par lequel Mme d'Aulnoy signait ses œuvres. L'auteur ainsi en quête de personnage se montre donc bel et bien comme vivant au cœur d'une société, dans l'orbite de la Cour et dotée de puissants protecteurs. Dans cet autoportrait, le personnage de Madame D. apparaît d'ailleurs au cœur de son époque et fait un éloge assez dithyrambique de la Cour de Louis XIV et de ses fastes. Les vers prononcés par l'entremise de la nymphe sont non seulement un compliment destiné à la princesse Palatine mais aussi un véritable panégyrique du règne :

Quand ce palais superbe et ces jardins tranquilles

Souvent de sa pompeuse Cour

Sont les agréables asiles,

De tout ce qui s'offre à vos yeux,

Est-il rien qui doive surprendre

Et ne devrait-on pas s'attendre

A voir tant de trésors enrichir ces beaux lieux ?...(vol. 1, p. 295)

On reconnaît ici l'attitude courtisane sans doute inévitable à l'époque. Le poème sent l'exercice d'école mais nul écrivain n'y échappait alors. Compte tenu du système des pensions, Molière lui-même dut se plier à la norme et faire l'éloge obligé de Louis XIV, le pouvoir considérant d'ailleurs cette publicité comme une sorte de contrat obligé. Cependant, en cette fin de siècle, le temps des largesses et des pensions est révolu, mais pas celui des grâces et des recours en grâce. Mme d'Aulnoy, à cause de sa vie tumultueuse, en avait bien besoin. De plus, la glorification du siècle de Louis Le Grand est aussi une marque d'appartenance au clan des Modernes. La nymphe de Saint-Cloud, bien proche d'une fée dans sa description même, est présentée avec « des yeux doux et brillants, l'air enjoué et spirituel, les manières gracieuses et polies (p. 294) ». Il s'agit pour le moins d'une nymphe revue et corrigée selon les normes de la beauté et des bienséances du siècle avec un parti-pris d'excellence dans le portrait qui la rapproche de nos contes. De plus cette nymphe fait ostensiblement l'éloge du siècle nouveau et du monarque lui-même. L'allusion au Roi-Soleil est d'ailleurs explicite :

Jamais un importun nuage

du Soleil en ces lieux ne couvre le visage. (vol. 1, p. 295)

Cette référence à peine voilée au roi permet à l'écrivain de vanter la beauté des lieux et le pouvoir de Louis XIV. La comparaison avec le monde de la fable antique tourne au détriment de cette dernière et à l'avantage d'une civilisation d'inspiration moderne et nationale, la nymphe s'écriant par exemple :

On cesse de vanter en voyant des beaux lieux

Les retraites délicieuses

Qu'habitaient autrefois les dieux. (vol. 1, p. 295) 
Madame D., alias Mme d'Aulnoy se situe ainsi dans la mouvance de Charles Perrault et des Modernes, avec la ferme résolution de faire l'éloge d'un siècle et d'une civilisation considérés comme incomparables surtout aux yeux du monarque absolu. Mme d'Aulnoy participe donc pleinement au panégyrique d'un règne considéré comme l'apogée de toute culture et de toute civilisation et supérieur à l'Antiquité. Les contes eux-mêmes en portent parfois la trace. "La Biche au bois» en est un exemple privilégié : le récit, par l'intermédiaire de la description du palais, fait l'éloge du pouvoir en place. On assiste en l'occurrence à une sorte de mise en abyme de l'histoire même du règne de Louis XIV. Le palais est en effet décoré par les fées, lesquelles avaient en guise de décoration retracé sur leurs tentures les actions du plus grand des rois. « Elles avaient pris pour le bâtir l'architecte du Soleil. Il avait fait en petit ce que celui du Soleil est en grand. » (vol. 2, p. 90). Cette appellation et cette référence à l'astre solaire évoquent bien entendu Louis XIV en personne et l'on trouve ensuite inséré dans le conte, sans autre préambule et sans lien narratif direct, sinon descriptif, l'éloge en vers du monarque qui se présente ainsi :

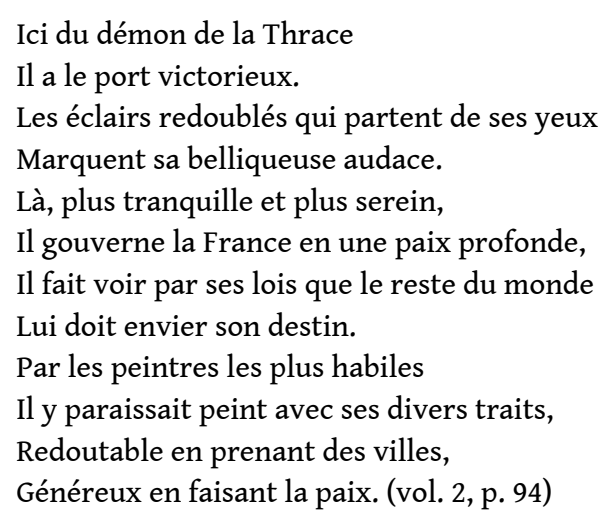

Ce panégyrique marque une fois de plus l'appartenance de notre conteuse au groupe des Modernes. L'analogie évidente, à travers l'image des éclairs qui partent des yeux, entre Jupiter et Louis XIV, tourne inexorablement à l'avantage de ce dernier. La fable antique n'est utilisée que pour mieux servir l'entreprise de glorification du siècle de Louis le Grand, par le style hyperbolique. Mais le double champ lexical de la guerre et de la paix a de quoi laisser perplexe le lecteur moderne. Lorsque l'on songe aux guerres incessantes de la fin du règne, à la ruine du pays et à la misère du peuple, on est quelque peu gêné par le panégyrique de ce Zeus moderne, digne du plus dévoué des courtisans. Mais pour le lecteur du $\mathrm{xVII}^{\mathrm{e}}$ siècle, il s'agissait là d'une marque d'appartenance à une classe sociale et à un statut d'écrivain vivant dans l'orbite de la Cour, dont les références culturelles elles-mêmes étaient liées à la volonté de gloire nationale du monarque. "La Biche au bois » contient même une allusion directe au mariage du duc de Bourgogne, petit-fils du Roi-Soleil, avec Marie-Adélaïde de Savoie, alors âgée de douze ans ; le conte lui sera dédié indirectement par l'insertion d'un petit poème lors de la description du personnage de la princesse avant sa métamorphose en biche :

Autour d'elle, on voyait les grâces

Les ris, les plaisirs et les jeux,

Et les Amours respectueux

Empressés à suivre ses traces ;

Elle avait l'air majestueux

Avec une douceur céleste.

Elle s'attirait tous les vœux 
Sans compter ici tout le reste.

Elle avait les mêmes attraits

Qui fit briller Adélaïde,

Quand l'hymen lui servant de guide

Elle vint dans ces lieux pour cimenter la paix. (vol. 2, p. 106)

De manière surprenante, les obligations du courtisan font oublier à Mme d'Aulnoy le thème habituel des mariages forcés. Il s'agit pourtant bien en l'occurrence d'un mariage arrangé à des fins politiques, concernant une très jeune fille, presque une enfant, devenue la coqueluche de la Cour en cette fin de règne. Le féminisme habituel de notre conteuse qui dénonce volontiers les mariages forcés courbe l'échine devant les contraintes sociales des allées du roi. L'éloge se fait cette fois d'une manière autre que par la référence à la supériorité des Modernes sur les Anciens. L'auteur met en parallèle réalité et fiction. C'est la fiction qui, de manière assez paradoxale, rejoint la réalité. À l'intérieur même des contes, on trouve donc trace d'une attitude courtisane de la part de Mme d'Aulnoy, attitude qui confirme l'appartenance au groupe privilégié des mondains vivant dans l'orbite de la Cour, mais aussi la nécessité d'un recours en grâce et de hautes protections liées à une vie quelque peu scandaleuse.

On pourrait citer d'autres exemples de cette attitude courtisane qui envahit l'univers de le féerie, lequel est le plus souvent un miroir de Versailles. L'opération de propagande la plus remarquable est cependant celle menée par Préchac. Celui-ci publie en 1698 à son tour un recueil intitulé Contes moins contes que les autres ${ }^{2}$. Dans ce recueil dont le titre même laisserait entendre que la réalité peut rejoindre la fiction, voire la dépasser, l'un des récits, "Sans Parangon " est une véritable allégorie dont l'objectif essentiel est la louange du roi. Le jeune prince Sans Parangon vit donc jusqu'à l'âge de vingt et un ans sous la protection de la fée Clairance et sous l'influence de Belle-Gloire à qui il s'efforce de ne jamais déplaire. Évidemment l'œuvre de Préchac reste une œuvre courtisane, bien que le fait de présenter un roi parfait puisse aussi être éventuellement perçu comme une critique indirecte du modèle en place.

9 Cependant au-delà de cette apparente flagornerie, le conte se prête à d'autres jeux plus subversifs et laisse place à une remise en cause d'un pouvoir ébranlé par la Fronde et la régence. Le miroir valorisant peut aussi devenir un miroir déformant, voire caricatural de la fin d'un règne. Il devient même, sous la plume des conteuses et de Mme d'Aulnoy en particulier, une forme plus sournoise de critique du pouvoir susceptible d'échapper à la censure. À ce titre l'œuvre de Mme d'Aulnoy est particulièrement révélatrice. Parallèlement à l'attitude courtisane, à l'éloge du pouvoir royal et à un aristocratisme affiché, ses contes et ses récits-cadres laissent place à une remise en cause de l'organisation de cette société de la fin $\mathrm{du}_{\mathrm{XvII}}^{\mathrm{e}}$ siècle dans une perspective que l'on pourrait qualifier de réformiste. D'autres œuvres contemporaines des contes, telles que le Télémaque de Fénelon ou Les Portraits de La Bruyère, laissent aussi entrevoir une volonté de changement au sein de l'organisation sociale, sans pourtant impliquer de véritables bouleversements révolutionnaires. On trouve ainsi dans les contes de Mme d'Aulnoy une critique insidieuse du pouvoir absolu et de sa relative fragilité. Les premières lignes de "Finette Cendron", récit qui amalgame le thème du "Petit Poucet » et de "Cendrillon » sont plutôt subversives :

Il était une fois un roi et une reine qui avaient mal fait leurs affaires. On les chassa de leur royaume. Ils vendirent leurs couronnes pour vivre, puis leurs habits, leurs linges, leurs dentelles et tous leurs meubles, pièce à pièce. Les fripiers étaient las d'acheter, car tous les jours ils vendaient chose nouvelle. Quand le roi et la reine furent bien pauvres, le roi dit à sa femme : "Nous voilà hors de notre royaume, 
nous n'avons plus rien, il faut gagner notre vie et celle de nos pauvres enfants; avisez un peu ce que nous avons à faire, car jusqu'à présent je n'ai su que le métier de roi qui est fort doux. » (vol. 1, p. 363)

10 Certes, l'incompétence royale est ici traitée de manière comique, mais pour le moins corrosive si l'on songe au pouvoir absolu qui caractérisait le siècle et l'absence de remise en cause possible de la souveraineté de droit divin. Le coup de griffe dans la satire sociale se prolonge d'ailleurs quelques lignes plus loin quand les filles des souverains sont traitées de franches paresseuses qui croient être de grandes dames. Souvenons-nous cependant que la Fronde est encore présente dans les esprits et surtout dans celui de Louis XIV. Or le thème de la sédition se trouve dans plusieurs contes. Dans "L'Oiseau bleu ", c'est bien un coup d'État qui permet à Florine de sortir de sa geôle après la mort du roi.

La fortune de la méchante reine et de sa fille changea de face; elles étaient regardées comme des favorites qui avaient abusé de leur faveur. Le peuple mutiné courut au palais demander la princesse Florine, la reconnaissant pour souveraine. La reine, irritée, voulut traiter l'affaire avec hauteur, elle parut sur le balcon et menaça les mutins. En même temps, la sédition devint générale; on enfonce les portes de son appartement, on le pille et on l'assomme à coups de pierres. (vol. 1, p. 101)

11 L'allusion aux favorites est assez scabreuse pour l'époque si l'on songe au contrepouvoir que représentaient Mme de Montespan ou Mme de Maintenon. Cependant, il faut noter aussi qu'il ne s'agit pas en l'occurrence d'une véritable révolution mais d'un retour à l'ordre initial, une sorte de restauration du pouvoir légitime d'ailleurs entérinée par les Grands du royaume qui s'assemblent promptement et couronnent l'héritière. L'instabilité du pouvoir est pourtant présente encore dans «La Princesse Carpillon », instabilité due d'abord aux luttes pour la succession mais aussi à la sédition à travers les aventures du berger Sublime qui, nous dit-on, « avait été roi d'un beau et florissant royaume ; un usurpateur, son voisin et son ennemi, conduisit heureusement ses intrigues secrètes et gagna certains esprits remuants qui se soulevèrent et lui fournirent les moyens de surprendre le roi et toute sa famille.» (vol. 2, p.1-56) Ces esprits remuants rappellent étrangement ces nobles frondeurs obligeant le jeune Louis XIV à fuir. Les événements de la Fronde, bien que se situant historiquement environ cinquante ans avant la parution des contes, semblent avoir profondément marqué les esprits et exercé encore une certaine influence, influence sans doute d'autant plus sensible que la fin du siècle correspond aussi à une fin de règne. Cette fragilité du pouvoir est d'ailleurs analysée de manière plus perspicace encore par notre conteuse, comme inhérente à un monde où seule compte l'apparence plus encore que la réalité. «L'Oiseau bleu » est assez révélateur à ce sujet. L'enchanteur met en garde le roi Charmant alors métamorphosé en ces termes :

Tel qui veut obéir à un homme ne veut pas obéir à un perroquet; tel vous craint étant roi, étant environné de grandeur et de faste, qui vous arrachera toutes les plumes vous voyant un petit oiseau. (vol. 1, p. 99)

12 Critique déjà fort insidieuse d'un pouvoir qui n'est lié qu'à l'apparence, donc susceptible d'être controversé, et non à une souveraineté de droit divin. Cependant la critique est atténuée par le ton plaisant avec lequel Mme d'Aulnoy traite les malheurs du roi Charmant métamorphosé en oiseau bleu :

Le clou qui l'accrochait s'était rompu, la cage était tombée et sa majesté emplumée souffrit beaucoup de cette chute; Minet, qui se trouvait dans la chambre lorsque cet accident arriva, lui donna un coup de griffe dans l'œil, dont il pensa rester borgne. 
Une autre fois, on avait oublié de lui donner à boire; il allait le grand chemin d'avoir la pépie, quand on l'en garantit par quelques gouttes d'eau. Un petit coquin de singe s'étant échappé attrapa ses plumes au travers des barreaux de la cage et il l'épargna aussi peu qu'il l'aurait fait d'un geai ou d'un merle. Le pire de tout cela c'est qu'il était sur le point de perdre son royaume; ses héritiers faisaient tous les jours des fourberies nouvelles pour prouver qu'il était mort. (vol. 1, p. 102)

Une expression telle que «sa majesté emplumée » manifeste quelque irrévérence de la part de notre conteuse mais le ton plaisant, joint à l'humour, atténue fortement la satire du pouvoir. Le problème de la succession reste cependant posé à la fin de cette citation et celui-ci revient dans d'autres récits. Ainsi se dévoile dans les contes de Mme d'Aulnoy une satire, certes discrète et plaisante, mais cependant fréquente, de la fragilité du pouvoir absolu.

Mais la critique peut être plus profonde. Quelques réflexions concernant l'arbitraire du pouvoir émaillent aussi les récits. Le héros de "La Belle aux cheveux d'or ", à l'image du surintendant Fouquet, se retrouve incarcéré sur simple délation, traîné en prison, sans autre forme de procès, avec un peu de paille pour se coucher et condamné à mourir de faim. Georges Mongrédien ${ }^{3}$ nous décrit d'une manière précise le caractère arbitraire des emprisonnements au $\mathrm{XVII}^{\mathrm{e}}$ siècle et le traitement fort différencié des prisonniers, en particulier en ce qui concerne les victimes ordinaires des lettres de cachet, soustraites à toute juridiction et qui ne pouvaient attendre leur élargissement que $\mathrm{du}$ bon vouloir du roi et des ministres. «La Princesse Carpillon» contient une allusion plus discrète encore à certaines pratiques pour le moins discutables du pouvoir absolu de l'époque : incarcération, tortures, exécutions, où le sadisme des spectateurs était cultivé. La fée indique en effet aux bergers qu'elle vient de délivrer du centaure, symbole ici de la tyrannie, que "la justice et la raison s'opposent à des coutumes si barbares » (vol. 2, p. 11). Jacques Barchilon ${ }^{4}$ suggère d'ailleurs que cette affirmation est une remise en cause des coutumes judiciaires de l'époque.

On trouve de même une allusion aux guerres incessantes et au système de conscription dans les premières lignes de "Belle-Belle et le chevalier Fortuné ». On y voit un roi dépouillé par un rival plus puissant rassembler « quelques troupes dont il composa une petite armée et pour la grossir en peu de temps, il fit publier une ordonnance par laquelle il voulait que tous les gentilshommes de son royaume vinssent la servir en personne ou lui envoyassent un de leurs enfants qui fussent bien équipés d'armes et de chevaux et disposés à seconder toutes ses entreprises. » (vol. 2, p. 216) On retrouve bien là le lien d'allégeance encore existant au XVII siècle entre la noblesse et son suzerain, mais la suite du texte est plus intéressante encore quant à l'évolution de ces problèmes de recrutement de soldats à la fin du règne de Louis XIV. Le personnage qui reçoit l'ordre d'aller défendre le royaume est un vieillard qui n'est plus apte physiquement au service. Il devrait donc verser une très grosse amende et se désole de n'être point en état de payer la taxe. Or la critique ainsi portée indirectement par notre conteuse vise une cible bien précise à l'époque. C'est en effet au tout début $d u x{ }^{e}{ }^{e}$ siècle que le pouvoir royal entérine l'achat de remplaçants pour le service par le paiement d'une taxe afin de renflouer le trésor royal. On trouve des critiques tout aussi ciblées cependant dans "La Chatte blanche " où la bonté du cœur est évoquée comme une marchandise rare parmi les princes qui «veulent être aimés de tout le monde et ne veulent rien aimer. » (vol. 2, p. 179).

16 Au-delà de ces coups de griffe portés au pouvoir absolu de Louis XIV, pouvoir absolu déjà sur le déclin en cette fin de règne, certaines analyses présentées sous forme de 
remarques moralisantes peuvent même être perçues comme une remise en question de l'ordre établi. C'est ainsi que le héros de " La Belle aux cheveux d'or » délivre le corbeau des serres de l'aigle en remettant en question la loi du plus fort :

Voilà, dit-il, comme les plus forts oppriment les plus faibles; quelle raison a l'aigle de manger le corbeau ? (vol. 1, p. 61)

17 On croit retrouver des accents pascaliens dans cette évocation des rapports qui s'instaurent entre justice et force, mais ce qui est nouveau et pour le moins subversif, c'est qu'Avenant le héros joue le rôle de libérateur des opprimés et tue l'aigle, dont on sait qu'au niveau symbolique il représente le pouvoir absolu. Dans le conte «Le Dauphin ", l'analyse est plus osée encore. La parabole atténue à peine la critique et c'est toute une remise en cause de la société qui est ici suggérée. Le dauphin s'écrie en effet :

Quoi, vous êtes hommes et vous vous étonnez de l'injustice des hommes ? En vérité, vous n'y pensez point, c'est tout ce que vous pourriez faire si vous étiez poisson; encore ne sommes-nous pas trop équitables dans notre empire salé et l'on voit tous les jours les plus gros qui engloutissent les plus petits ; on ne devrait pas le souffrir, car le moindre hareng a son droit de citoyen acquis dans la mer aussi bien qu'une affreuse baleine. (vol. 2, p. 498)

Ce hareng réclamant l'égalité des droits n'annonce-t-il pas le xviII siècle et la révolution? L'affirmation, bien que transposée dans une parabole, est pour le moins subversive. La notion même de citoyen semble sonner le glas d'une société inégalitaire. C'est donc une remise en cause de toute une organisation sociale qui se trouve ainsi exprimée.

Mme d'Aulnoy critique d'ailleurs non seulement le pouvoir royal mais aussi le monde de la Cour, monde de flatterie et d'apparence dont l'hypocrisie elle-même minimise la culpabilité des princes. Le Prince Marcassin, héros éponyme du conte, dans un dialogue avec sa mère, souligne les problèmes ainsi posés par les rapports de Cour car, malgré son aspect monstrueux, il constate :

Je ne vois personne qui ne me loue et qui ne me fasse apercevoir que j'ai mille bonne qualités.

- Tels sont les courtisans, dit la reine, et telle est la condition des princes. Les uns

louent toujours et les autres sont toujours loués. (vol. 2, p. 449)

Telle était aussi probablement la Cour de Versailles; cependant aux yeux de Mme d'Aulnoy, la responsabilité de ce jeu truqué dans les relations sociales revient aussi bien au monarque qu'aux courtisans. Quand la reine poursuit le dialogue précédent, c'est en effet pour affirmer que les Grands seraient plus heureux s'ils avaient des amis plus attachés à leur personne qu'à leur fortune mais le prince réplique alors que les vérités désagréables sont difficiles à accepter et que de quelque condition qu'on soit, on ne les aime pas. Une critique similaire se retrouve dans le conte intitulé «La Princesse Belle-Étoile et le Prince Chéri ». Les corsaires qui ont recueilli les jeunes gens refusent de les accompagner à la Cour où règnent « des agitations continuelles, des soins, des déguisements, des feintes, de l'envie, des inégalités, des véritables maux et de faux biens. » (vol. 2, p. 363) Critique assez virulente du monde de l'apparence qui représente de manière à peine voilée la Cour de Louis XIV à travers ce champ lexical de la fausseté. Il s'agit là d'une description pour le moins subversive d'une société vivant dans l'orbite du souverain, société où règne la compétition pour la possession de quelques privilèges. Monde étrange en effet que celui de la Cour de Louis XIV dont tous les historiens s'accordent à démonter le système comme celui d'une prise de pouvoir d'un roi et d'une autodestruction consentie de toute la classe aristocratique. 
21 De manière surprenante et pour le moins ambiguë, on trouve donc chez notre conteuse, parallèlement au panégyrique du siècle de Louis XIV contenu dans les dédicaces et inséré parfois dans les contes, une sorte de remise en cause de cette civilisation et de cette suprématie du Grand Siècle. Mais il est une forme particulière sous laquelle se présente parfois cette subversion liée à l'art de la bagatelle, celle qui consiste, sous prétexte de merveilleux, à peindre un autre monde organisé différemment et susceptible de mener au bonheur. On trouve ainsi dans les contes de Mme d'Aulnoy les premiers balbutiements du mythe du Bon Sauvage. Mme d'Aulnoy fait à plusieurs reprises dans les contes preuve d'un certain esprit de résistance à l'égard d'une civilisation moderne qu'elle présente comme pervertie par rapport à l'antique simplicité d'une vie rustique. Cet éloge d'une vie proche de l'état de nature est une sorte de remise en question de l'excellence même du Grand Siècle. Alors que la civilisation de Louis le Grand doit apparaître comme la référence absolue, on trouve aussi, mis en valeur comme en une sorte de contrepoint, le retour à un état proche de l'état de nature. On constate à plusieurs reprises cette notion d'écart dans l'œuvre de la conteuse par rapport à la norme des valeurs établies, écart qui correspond bien à une époque de crise de la conscience européenne qui se manifestera quelques années plus tard dans la contre-société des Troglodytes illustrée par Montesquieu dans les Lettres Persanes. Le thème de l'enfant sauvage correspond certes à une réalité historique et sociale mais il apparaît à deux reprises largement développé dans les contes comme une sorte d'idéal naturel. Dans «L'Oranger et l'abeille », c'est par des ogres vivant dans des grottes et dans une atmosphère proche des Troglodytes qu'est élevée la princesse Aimée :

Elle ne savait ni lire, ni écrire, ni aucunes langues, elle parlait le jargon d'Ogrelie ; elle vivait dans une parfaite ignorance de toutes les choses du monde; elle ne laissait pas d'avoir d'aussi bons principes de vertu, de douceur et de naturel que si elle avait été élevée dans la Cour de l'univers la mieux polie. (vol. 1, p. 247-248)

Cette Cour de l'univers la mieux polie n'est pas sans rappeler Versailles et se trouve ainsi mise sur un pied d'égalité avec un état naturel et sauvage, ce qui représente un écart certain par rapport aux panégyriques habituels du Grand Siècle, considéré comme une sorte d'apogée de l'évolution de l'humanité. Mme d'Aulnoy remettrait ainsi en cause la doxa de l'époque. Ce principe d'égalité devient même un rapport de supériorité dans la suite du texte. La jeune sauvageonne surpasse donc les nobles dames évoluant dans l'orbite de la Cour. Le même thème est traité de nouveau dans «La Princesse Carpillon ». Ce récit débute par l'abandon d'un nourrisson qui se trouve recueilli par des aigles puis par des bergers. Au-delà de la référence culturelle sous-jacente à la louve romaine, l'épisode apparaît de nouveau comme un prétexte à l'éloge d'une vie rustique proche de l'état naturel et considérée comme supérieure même à l'état civilisé.

Elle (l'aiglonne) exerça mieux le droit d'hospitalité que bien des gens ne le savent

faire. Elle se mit proche de son nourrisson, elle étendit ses ailes et le réchauffa, il sembla que tous ses soins n'étaient plus que pour lui; un instinct particulier l'engagea d'aller chercher des fruits, de les becqueter et d'en verser le jus dans la bouche vermeille du petit prince; enfin elle le nourrit si bien que la reine sa mère n'aurait su le nourrir mieux. (vol. 2, p. 6)

Les comparatifs privilégient ici l'espèce animale comme plus proche d'un état naturel. Cette supériorité est d'ailleurs de nouveau mise en valeur par une petite remarque contenue dans «Le Prince Marcassin » qui, en tant qu'être ambivalent, mi-homme mianimal, s'écrie : 
Ce défaut de parole que vous me reprochez, cette petite finesse où je ménage mes intérêts, c'est justement l'homme qui agit; car, à vous parler sans façon, les animaux ont plus d'honneur entre eux que les hommes. (vol. 2, p. 463) rappelle étrangement l'œuvre de Calderon de la Barca, El Gran teatro del mundo, où l'on voit un personnage saltimbanque, le sieur Monde, mettre en scène des pièces où tout n'est qu'apparence et fiction. Cette remise en cause métaphysique d'un rôle importun sur le grand théâtre du monde, remise en cause d'un pouvoir fondé sur un monde de duperie et de fausseté apparaît en contradiction avec les panégyriques du Roi Soleil ou du siècle de Louis le Grand pourtant aussi présents dans l'œuvre de Mme d'Aulnoy. L'attitude de celle-ci à l'égard du pouvoir absolu en place parait donc pour le moins fort ambiguë. Derrière la soumission apparente de la comtesse d'Aulnoy et la courtisanerie quasi-obligée pour tout écrivain de l'époque pourrait bien se cacher une écriture plus subversive remettant en cause l'excellence même du règne et annonçant la critique des philosophes du XviII ${ }^{\mathrm{e}}$ siècle. Notre conteuse quitterait donc aisément l'allée du roi pour s'égarer sur les sentiers à peine tracés du Bon Sauvage et le miroir de la Cour deviendrait dès lors un miroir déformant qui renvoie un reflet moins valorisant à ceux qui s'y mirent.

Dans le parcours sur cette carte du Pouvoir, il est d'ailleurs un lieu imaginaire qui cristallise ce double reflet : c'est celui de l'île. Le thème de l'île merveilleuse est certes traditionnel mais il devient chez certains conteurs de cette fin de siècle, l'occasion de peindre une forme de société idéale, sorte d'anti-monde ou de nouveau monde qui implique une critique à peine voilée du monde réel. La fiction devient un double 
idéalisé, expression subversive d'un rêve plus ou moins accessible. C'est en cette époque charnière entre le $\mathrm{xVII}^{\mathrm{e}}$ et le $\mathrm{xVIII}^{\mathrm{e}}$ siècle que l'on voit fleurir toute une série d'ouvrages qui développent le thème de l'île comme un lieu de L'Utopie de Thomas More. Les conteurs et les conteuses de cette fin du XVII siècle ont pu être influencés par ce courant utopiste, proche au demeurant du courant libertin auquel beaucoup appartenaient. "L'île inaccessible» du chevalier de Mailly, contemporain de Mme d'Aulnoy, en est un exemple.

29 À la fin des Illustres fées se situe ce récit de «L'Île inaccessible ${ }^{5}$ ». La vie parfaitement heureuse dans l'île se distingue de l'évocation des autres pays merveilleux dont il est toujours suggéré que l'on désire à un moment donné en sortir. Mais ce qui est plus intéressant, c'est le thème de l'exclusion des étrangers et la peinture des mœurs innocentes des îliens. La supériorité de l'humanité primitive est esquissée au détriment d'une forme de vie sociale qui est présentée comme soumise aux tracas engendrés par la civilisation. Il s'agit bien d'évoquer en quelques lignes un bonheur idyllique dans une nature généreuse, dans une sorte de paradis originel protégé des intrusions de l'homme civilisé.

Les princes de l'île, depuis un siècle ou deux, avaient eu la curiosité de savoir ce qui se passait en terre ferme, et leur coutume était d'envoyer de temps en temps des espions chez leurs plus proches voisins. Ils y envoyaient les plus affidés et les plus habiles de leurs courtisans à qui les fées donnaient par leur art, le pouvoir de voler aussi loin qu'il leur plaisait, en se reposant de temps en temps sur quelque rocher. Elles leur avaient aussi donné le don de devenir invisibles, en leur faisant porter des robes qui étaient brillantes comme la lumière du jour. Cette commodité d'envoyer chez les voisins avait instruit les habitants de l'île de tout ce qui se passait dans le monde, si bien qu'il s'était élevé parmi eux des troupes de politiques, ou autrement des nouvellistes qui raisonnent comme leurs pareils raisonnent à Paris sur les desseins et la conduite des potentats. (p. 170-171)

Ce portrait des habitants de "l'île inaccessible» reprend certes les habituelles techniques de création d'un univers merveilleux en évoquant les voyages aériens qui sont monnaie courante dans les récits féeriques. De manière paradoxale, dans le récit du chevalier de Mailly, les habitants de l'île utilisent ce don pour espionner ce qui se passe dans l'autre monde. Le renversement des données est intéressant. Il ne s'agit pas seulement d'esquisser le mythe du Bon Sauvage mais de dépeindre une contre-société tout aussi développée que l'autre mais qui aurait su évoluer de manière positive. Les troupes de politiques, de nouvellistes sont les précurseurs des philosophes du siècle des Lumières. Le thème de l'insularité se révèle donc plus subversif qu'il n'y parait au premier regard et la description de ces îles merveilleuses se transforme en une critique à peine voilée d'une fin de règne marquée par les guerres incessantes et la ruine d'un pays.

31 Alors que Perrault ne traite jamais de l'insularité, ce n'est sans doute pas un hasard si Mme d'Aulnoy qui lance la mode des récits féeriques décline elle aussi mais au féminin le topos de l'île merveilleuse. L'insula feminarum apparaît dans ses contes comme la terre promise par excellence, espace de conquête ou à conquérir pour remplacer un monde où le pouvoir se décline exclusivement au masculin. Si la notion d'écart par rapport à la norme sociale de l'époque apparaît dans les premiers balbutiements du thème $d u$ Bon Sauvage, elle rejoint à travers le thème de l'île gouvernée par des femmes une autre forme de contestation d'un ordre social établi. Le mythe du pays interdit aux hommes, pays où règne un bonheur régi par de fières amazones, est évoqué dans « Le Prince Lutin ». Les fées sont d'ailleurs présentées dans 
ce conte comme échappant au pouvoir masculin. L'amour masculin est ainsi décrit comme une rupture de l'ordre social établi et entraîne par conséquent le déséquilibre. Il s'ensuit une sorte de rétablissement d'un ordre initial perturbé par la présence masculine. La fée déçue recrée un royaume d'où l'homme est à tout jamais banni :

Dès qu'elle fut de retour, elle transporta son palais, elle en chassa les gardes et les officiers; elle prit des femmes de race d'amazones; elle les envoya autour de son île pour y faire une garde exacte afin qu'aucun homme n'y pût entrer. Elle nomma ce lieu "l'île des plaisirs tranquilles »; elle disait toujours qu'on n'en pouvait avoir de véritables quand on faisait quelque société avec les hommes; elle éleva sa fille dans cette opinion. (vol. 1, p. 133)

Ce pays sans hommes reprend à la fois le thème des amazones hérité de la fable antique et la recherche d'un bonheur parfait qui sera développé au xviII siècle par l'évocation d'un lieu d'autosuffisance et de rupture avec le reste du monde. Ce thème du pays d'où les hommes sont bannis réapparaît de nouveau à la fin du conte L'Oranger et l'Abeille :

Le bois où l'oranger était servait de promenade à une princesse qui demeurait dans un palais magnifique; elle avait de la jeunesse, de la beauté et de l'esprit. On l'appelait Linda. Elle ne voulait point se marier parce qu'elle craignait de n'être pas toujours aimée de celui qu'elle choisirait pour époux et comme elle avait de grands biens, elle fit bâtir un château somptueux et elle n'y recevait que des dames et des vieillards plus philosophes que galants, sans permettre qu'aucuns autres cavaliers en approchassent. (vol. 1, p. 270)

Ce personnage doué de jeunesse, de beauté et d'esprit, correspond étrangement au portrait que les contemporains dressent de notre conteuse et l'idéal de cette vie salonnière excluant l'homme en tant que tel pourrait bien être à l'image même d'un idéal de société rêvé par Mme d'Aulnoy. Mme d'Aulnoy va au-delà de l'évocation de cette supériorité féminine éventuelle dans les rapports amoureux : elle suggère même la possibilité d'un pouvoir politique qui pourrait être l'apanage du sexe faible. Les femmes détenant le pouvoir sont, en effet, assez nombreuses dans les contes. BelleBelle alias le chevalier Fortuné que nous avons déjà évoquée en est un excellent exemple. Cette jeune fille qui part faire la guerre, déguisée en homme, rappelle, au-delà de l'image du travesti, la grande Mademoiselle et d'autres héroïnes de la Fronde, encore bien vivantes dans la mémoire de Louis XIV et des contemporains du grand siècle. Elle devient grand écuyer du roi qui prend conseil auprès d'elle. Roi assez falot au demeurant, car le pouvoir appartient en fait à la reine-sœur, reine douairière qui exerce une sorte de droit d'aînesse sur son frère. La revendication féministe est ici tangible au niveau politique. Il arrive fréquemment que les femmes règnent dans l'univers de nos contes. C'est le cas de La Belle aux cheveux d'or ou de Florine, l'héroïne de «L'Oiseau bleu», que le peuple porte d'ailleurs au pouvoir après une mutinerie dirigée contre d'autres femmes, en l'occurrence la marâtre et sa fille. La Chatte blanche agit aussi en souveraine absolue, menant la chasse à courre, rendant justice contre des conspirateurs et traitant d'égal à égal et même dans un rapport de supériorité avec le vieux roi qui refuse d'abandonner le pouvoir : image osée de la fin d'un règne.

La puissance suprême accordée aux fées, puissance supérieure au pouvoir des hommes, n'est-elle pas aussi révélatrice d'un certain féminisme latent dans l'œuvre de Mme d'Aulnoy? Il est d'ailleurs significatif que les magiciens pourtant présents dans la tradition pastorale qui influencera Mme d'Aulnoy cèdent la place aux fées dans les contes. Les enchanteurs y sont rares et quand ils apparaissent, leur pouvoir est présenté comme largement inférieur à celui des dames blanches. Ces puissances qui se présentent sous une apparence toute féminine ne sont d'ailleurs pas toutes semblables 
à la fée Bénigne. La fée-amazone de "La Princesse Carpillon » a de quoi faire frémir. Elle commande aux rois et détient tous les pouvoirs, y compris le pouvoir judiciaire si bien qu'« elle fondit sur le coupable Bossu et lui portant un coup de sa lance ardente dans le cœur, elle ne lui laissa pas envisager longtemps les horreurs de la mort; il fut consumé comme s'il avait été brûlé par le tonnerre.» (vol. 2, p. 55) Le sadisme dans l'exécution capitale n'émeut guère notre conteuse qui semble même s'y complaire : il ne faut pas oublier qu'elle fut elle-même responsable de deux têtes tranchées en place de grève.

Le même féminisme virulent est d'ailleurs observable dans la nouvelle-cadre du Nouveau gentilhomme bourgeois. Non seulement les femmes y détiennent le pouvoir littéraire en tant que narrateurs, mais le personnage de la baronne de Saint-Thomas, maîtresse-femme et mère, a le monopole du pouvoir domestique. De plus, l'une de ses altercations avec le personnage de La Dandinardière est la démonstration d'une supériorité féminine revendiquée comme telle. Alors que le bourgeois gentilhomme joue les êtres cultivés et s'étonne que la baronne puisse comprendre le latin, celle-ci s'écrie :

Les femmes sont à présent aussi savantes que les hommes: elles étudient et sont capables de tout. C'est trop de dommage qu'elles ne puissent être dans les charges; un parlement composé de femmes serait la plus jolie chose du monde. (vol. 2, p. 374)

Ces propos traduisent une revendication profonde, celle de l'accès pour les femmes aux sphères officielles du pouvoir au même titre que les hommes. La suite du texte laisse d'ailleurs le lecteur perplexe ; la baronne conclut ainsi :

Se pourrait-il rien de plus agréable qu'une sentence de mort prononcée par une belle bouche bien incarnate et bien riante?

Cette belle bouche n'évoque-t-elle pas la baronne d'Aulnoy elle-même, une personnalité ambiguë, obligée de courber l'échine du moins en apparence mais capable d'une cruelle vengeance à l'encontre d'un pouvoir politique masculin symbolisé par un époux qu'elle s'efforce de faire périr en le dénonçant à la justice du roi ?

\section{NOTES}

1. On se référera à l'édition du tricentenaire en deux volumes dans cet article.

M.-C. d'Aulnoy, Contes I, Les contes des fées, introduction par Jacques Barchilon, texte établi et annoté par Philippe Hourcade, Paris, Société des textes Français modernes, Klincksieck, 1997, 604 p. et Contes II, Contes nouveaux ou les fées à la mode, 1998, 577 p.

Signalons cependant la réédition en cours d'un nouveau Cabinet des fées dont le premier volume est consacré à l'œuvre de Mme d'Aulnoy: Madame d'Aulnoy, Contes des fées suivis des Contes nouveaux ou Les Fées à la mode, édition critique par Nadine Jasmin, Paris, Champion, «Bibliothèque des Génies et des Fées », vol. 1, 2004, 1220 p.

2. Pour les recueils de contes autres que ceux de Mme d'Aulnoy, on se réfèrera au Cabinet des fées ou collection choisie des contes des fées et autres contes 
merveilleux ornés de figures, Amsterdam, 1785-1789, Genève, 41 volumes in-8. Préchac, Contes moins contes que les autres «Sans Parangon » et « La Reine des fées », Cabinet des fées, $t$. V in-8, p. 266-461.

3.G. Mongrédien, La Vie quotidienne sous Louis XIV, Paris, Hachette, 1948, 250 p.

4.J. Barchilon, Le Conte merveilleux Français de 1690 à 1790, cent ans de féerie et de poésie ignorées de l'histoire littéraire, Paris, Champion, 1975, p. 47.

5. Cabinet des fées, ouvr. cité, t. 5, p. 169 et suiv.

\section{RÉSUMÉS}

Les contes de Mme d'Aulnoy sont ancrés sociologiquement dans une époque, celle de la fin du règne de Louis XIV. À un premier niveau de lecture, les contes semblent n'être que le reflet valorisant du siècle du Roi-Soleil dont ils font le panégyrique. Mais ce miroir valorisant est aussi un miroir déformant qui renvoie à une mise en cause de ce pouvoir absolu. Il en sape les fondements et en conteste l'excellence en présentant comme un idéal le retour à une vie sauvage, proche de l'état de nature. De l'allée du roi, le lecteur est ainsi invité à passer de l'autre côté du miroir et à suivre le sentier à peine tracé du Bon Sauvage. Cet autre monde merveilleux parfois proche de la société des Troglodytes se présente aussi sous la forme d'une île et plus particulièrement chez la conteuse sous la forme d'une insula feminarum, société idéale où les femmes détiendraient enfin un pouvoir politique jusqu'alors réservé aux hommes.

From the King's Alley to the Paths of the Noble Savage: a Trajectory in Madame d'Aulnoy's Tales. Madame d'Aulnoy's tales are sociologically rooted at the end of Louis XIV's reign. At a first glance the tales seem to be but the image-enhancing reflection of the Sun-King, whose virtues they extol. But this mirror on the wall distorts the image we receive of the king and leads the onlooker to question the monarch's absolute power. It undermines its fondations and disputes its excellence, setting as an ideal the return to a simple existence, a state close to Nature. From the king's alley the reader is thus invited to go through the looking-glass and follow the trace of the path barely marked out by the "Noble Savage". This other wonderland, which sometimes resembles a troglodytes'society, is also presented as an island and, more particularly in the storyteller's mind as an insula feminarum, an ideal society in which women would, at last, hold a political power which had, until then, been reserved for men.

\section{AUTEUR}

MARIE-AGNÈS THIRARD

Université de Lille 3 